\title{
The Quantum Pair of Pants
}

\author{
Slawomir KLIMEK ${ }^{\dagger}$, Matt MCBRIDE ${ }^{\ddagger}$, Sumedha RATHNAYAKE ${ }^{\dagger}$ and Kaoru SAKAI ${ }^{\dagger}$ \\ $\dagger$ Department of Mathematical Sciences, Indiana University-Purdue University Indianapolis, \\ 402 N. Blackford St., Indianapolis, IN 46202, USA \\ E-mail:sklimek@math.iupui.edu,srathnay@iupui.edu,ksakai@iupui.edu \\ $\ddagger$ Department of Mathematics, University of Oklahoma, 601 Elm St., Norman, OK 73019, USA \\ E-mail: mmcbride@math.ou.edu
}

Received October 24, 2014, in final form February 03, 2015; Published online February 10, 2015

http://dx.doi.org/10.3842/SIGMA.2015.012

\begin{abstract}
We compute the spectrum of the operator of multiplication by the complex coordinate in a Hilbert space of holomorphic functions on a disk with two circular holes. Additionally we determine the structure of the $C^{*}$-algebra generated by that operator. The algebra can be considered as the quantum pair of pants.
\end{abstract}

Key words: quantum domains; $C^{*}$-algebras

2010 Mathematics Subject Classification: 46L35

\section{Introduction}

In this paper we study the operator $z$ of multiplication by the complex coordinate in Hilbert spaces of holomorphic functions on certain multiply connected domains in the complex plane. The domains we consider are disks with circular holes. The case of a disk with no holes is the classical one. In the Hardy space of the disk the multiplication operator $z$ is the unilateral shift whose spectrum is the disk. The $C^{*}$-algebra generated by the unilateral shift, the Toeplitz algebra, is an extension of the algebra of compact operators by $C\left(S^{1}\right), S^{1}$ being the boundary of the disk [4]. For the Bergman space the operator $z$ is a weighted unilateral shift and its spectrum and the $C^{*}$-algebra it generates are the same as in the Hardy space [7]. Partially for those reasons the Toeplitz algebra is often considered as the quantum disk [7, 9, 10].

A disk with one hole is biholomorphic to an annulus. In the Bergman space for example, the $z$ operator is a weighted bilateral shift with respect to the natural basis of (normalized) powers of the complex coordinate. Its spectrum is the annulus, and the $C^{*}$-algebra it generates is an extension of the algebra of compact operators by $C\left(S^{1} \times S^{1}\right)$, where $S^{1} \times S^{1}$ is the boundary of the annulus. The same is true for many other Hilbert spaces of holomorphic functions on an annulus. The resulting $C^{*}$-algebra is the quantum annulus of $[10,12]$.

In this paper we study in detail the two hole case: a pair of pants. Up to biholomorphism we can realize a disk with two holes as an annulus centered at zero with outer radius one, with an additional off centered hole. In the space of continuous functions on the closed pair of pants that are holomorphic in its interior, we consider a specific inner product with respect to which the operator of multiplication by the complex coordinate $z$ has a particularly simple structure. The results we obtain are completely analogous to zero and one-hole cases: the spectrum of $z$ is the domain of the corresponding pair of pants while the $C^{*}$-algebra generated by $z$ is an extension of the algebra of compact operators by $C\left(S^{1} \times S^{1} \times S^{1}\right)$, where $S^{1} \times S^{1} \times S^{1}$ is the boundary of the pair of pants.

This work is part of an ongoing effort to understand the structure of quantum Riemann surfaces and their noncommutative differential geometry, see $[7,8,10,11,12,13,14,15]$. Our 
paper has many things in common with the work of Abrahamse [1] and Abrahamse-Douglas [2], who use different Hilbert spaces.

The paper is organized as follows. Section 2 contains an overview of the zero and one-hole cases, while Section 3 has a detailed discussion of the quantum pair of pants.

\section{Preliminaries}

In this section we describe in some detail, the zero and one-hole cases. Most of the material is well-known, however the treatment of the quantum annulus is somewhat new.

\subsection{The quantum disk}

In this subsection we look at the structure of the quantum disk. We review the tools and the relevant theorems that will be a motivation for the subsequent discussion of the quantum pair of pants.

Consider the closed unit disk $\mathbb{D}=\{\zeta \in \mathbb{C}:|\zeta| \leq 1\}$. We can represent any holomorphic function inside the disk as a convergent power series

$$
f(\zeta)=\sum_{n=0}^{\infty} e_{n} \zeta^{n}
$$

The Hardy space on the disk is defined as

$$
H^{2}(\mathbb{D})=\left\{f(\zeta)=\sum_{n=0}^{\infty} e_{n} \zeta^{n}: \sum_{n=0}^{\infty}\left|e_{n}\right|^{2}<\infty\right\} .
$$

We define the multiplication operator by the complex coordinate, $z: H^{2}(\mathbb{D}) \rightarrow H^{2}(\mathbb{D})$ by the formula $f(\zeta) \mapsto \zeta f(\zeta)$. If $E_{n}=\zeta^{n}$ is the orthonormal basis on $H^{2}(\mathbb{D})$, then applying $z$ to the basis elements produces $z E_{n}=E_{n+1}$ for all $n \geq 0$, i.e., $z$ is the unilateral shift; moreover, we have the following formula for the adjoint operator to $z$

$$
z^{*} E_{n}=\left\{\begin{array}{lll}
E_{n-1} & \text { for } & n \geq 1 \\
0 & \text { for } & n=0
\end{array}\right.
$$

Now we consider the $C^{*}$-algebra generated by $z$. This well-known algebra is called the Toeplitz algebra, denoted by $\mathcal{T}$, and has also been termed the quantum (noncommutative) disk. This is (partially) based on the following standard results collected here with sketches of proofs which serve as a guideline for considerations in the next section.

Theorem 2.1. The norm of $z$ is 1 . The spectrum of $z$ is all of $\mathbb{D}$, i.e., $\sigma(z)=\mathbb{D}$.

Proof. The norm computation is straightforward. By the norm calculation it then follows that the spectrum is a closed subset of the unit disk. To illustrate that any $\lambda$ in the interior of $\mathbb{D}$ is an eigenvalue of $z^{*}$, take $f_{\lambda}(\zeta)=\sum_{n=0}^{\infty} \lambda^{n} \zeta^{n}$ and so

$$
z^{*} f_{\lambda}(\zeta)=\sum_{n=1}^{\infty} \lambda^{n} \zeta^{n-1}=\lambda \sum_{n=1}^{\infty} \lambda^{n-1} \zeta^{n-1}=\lambda \sum_{n=0}^{\infty} \lambda^{n} \zeta^{n}=\lambda f_{\lambda}(\zeta)
$$

Let $\mathcal{K}$ be the algebra of compact operators in $H^{2}(\mathbb{D})$. The next observation tells us how the commutator ideal of $\mathcal{T}$, and $\mathcal{K}$ are related. 
Theorem 2.2. The commutator ideal of $\mathcal{T}$ is the ideal of compact operators.

Proof. Since $\mathcal{T}$ is generated by $z$ and $z^{*}$, the commutator ideal of $\mathcal{T}$ is equal to the ideal generated by the commutator $\left[z^{*}, z\right]$. Note that $\left[z^{*}, z\right]=P_{E_{0}}$, the orthogonal projection onto the span of $E_{0}$. Since this one-dimensional projection is a compact operator, it follows that the commutator ideal of $\mathcal{T}$ is contained in $\mathcal{K}$. To prove the opposite inclusion we look at the following rank one operators: $E_{i j}(f)=\left\langle f, E_{i}\right\rangle E_{j}$. Notice that $E_{i j}=z^{j} P_{E_{0}}\left(z^{*}\right)^{i}$, hence those operators belong to the commutator ideal of $\mathcal{T}$. But every compact operator is a norm limit of finite rank operators, which in turn are finite linear combinations of $E_{i j}$ 's. This verifies that the commutator ideal of $\mathcal{T}$ contains $\mathcal{K}$.

In order to state the next result, first we introduce some more notation. We identify $H^{2}(\mathbb{D})$, the Hardy space on the unit disk, with the subspace of $L^{2}\left(S^{1}\right)$ spanned by $\left\{e^{i n x}\right\}_{n \geq 0}$. Also given a continuous function $f$ on the unit circle, we denote the multiplication operator by $f$ as $M_{f}$. Let $P: L^{2}\left(S^{1}\right) \rightarrow H^{2}(\mathbb{D})$ be the orthogonal projection onto $\operatorname{span}\left\{e^{i n x}\right\}_{n \geq 0}$, then define the operator $T_{f}: H^{2}(\mathbb{D}) \rightarrow H^{2}(\mathbb{D})$ by $T_{f}=P M_{f}$. The operator $T_{f}$ is known as a Toeplitz operator. Since $\left\|M_{f}\right\|=\|f\|_{\infty},\left\|T_{f}\right\| \leq\|f\|_{\infty}$ and hence it is bounded. We have:

Theorem 2.3. The quotient $\mathcal{T} / \mathcal{K}$ is isomorphic to $C\left(S^{1}\right)$, the space of continuous functions on the unit circle.

Proof. The usual proof constructs an isomorphism between the two algebras. Notice that for a continuous function $f$, we have $T_{f} \in \mathcal{T}$ and since $T_{e^{i x}}$ is the unilateral shift, $T_{e^{-i x}}=T_{e^{i x}}^{*}$. By the Stone-Weierstrass theorem, every continuous function can be approximated by trigonometric polynomials. Consequently we can define a map $\theta: C\left(S^{1}\right) \rightarrow \mathcal{T} / \mathcal{K}$ by $\theta: f \mapsto\left[T_{f}\right]$, the class of operators $T_{f}$.

Next we show that $T_{f}$ is compact if and only if $f \equiv 0$. Suppose $T_{f}$ is compact. Then for a continuous $f$ with Fourier series $\sum_{n=-\infty}^{\infty} e_{n} e^{i n x}$ we have

$$
T_{f}\left(e^{i k x}\right)=\sum_{n=0}^{\infty} e_{n-k} e^{i n x} .
$$

Thus, the matrix coefficients $e_{n}=\left(E_{i+n}, T_{f} E_{i}\right)$ and since $T_{f}$ is compact, we must have $\left(E_{i+n}\right.$, $\left.T_{f} E_{i}\right) \rightarrow 0$ as $i \rightarrow \infty$ for each fixed $n$. Therefore, $e_{n}=0$ for all $n$ and hence $f \equiv 0$. This result means that $\theta$ is injective.

Next we observe that $T_{f} T_{g}-T_{f g}$ is a compact operator for all continuous $f, g$. If $f, g$ are trigonometric polynomials then a direct calculation shows that $T_{f} T_{g}-T_{f g}$ is a finite rank operator. The general case then follows by appealing to the Stone-Weierstrass theorem. As a consequence, the map $\theta$ above is a $C^{*}$-homomorphism.

The range of $\theta$ is dense since it contains (the classes of) polynomials in $z$ and $z^{*}$. Then by general $C^{*}$-algebra theory (see [5] for example) $\theta$ is an isometry hence the range is closed. This means that $\operatorname{Ran}(\theta)=\mathcal{T} / \mathcal{K}$ and therefore $\theta$ is a $*$-isomorphism.

Note that from the last theorem we get a short exact sequence

$$
0 \rightarrow \mathcal{K} \rightarrow \mathcal{T} \rightarrow C\left(S^{1}\right) \rightarrow 0
$$

We can compare this to the short exact sequence for the classical disk

$$
0 \rightarrow C_{0}(\mathbb{D}) \rightarrow C(\mathbb{D}) \rightarrow C\left(S^{1}\right) \rightarrow 0
$$

where $C_{0}(\mathbb{D})$ are the continuous functions on the disk that vanish on the boundary. 


\subsection{The quantum annulus}

Let $0<r<1$ and consider the annulus

$$
A_{r}=\{\zeta \in \mathbb{C}: r \leq|\zeta| \leq 1\} .
$$

The classical uniformization theory of Riemann surfaces implies that every open annulus is biholomorphically equivalent to an annulus of the above form.

We can write any holomorphic function $\varphi(\zeta)$ on the interior of $A_{r}$ as the following convergent version of Laurent series

$$
\varphi(\zeta)=\sum_{n=0}^{\infty} e_{n} \zeta^{n}+\sum_{n=-\infty}^{-1} f_{n}\left(\frac{\zeta}{r}\right)^{n}
$$

We label the basic monomials in the above expansion as

$$
E_{n}=\zeta^{n}, \quad F_{n}=\left(\frac{\zeta}{r}\right)^{n}
$$

and define our specially convenient Hilbert space of holomorphic functions on $A_{r}$ to be

$$
H=\left\{\varphi(\zeta)=\sum_{n=0}^{\infty} e_{n} E_{n}+\sum_{n=-\infty}^{-1} f_{n} F_{n}:\|\varphi\|<\infty\right\},
$$

where

$$
\|\varphi\|^{2}=\sum_{n=0}^{\infty}\left|e_{n}\right|^{2}+\sum_{n=-\infty}^{-1}\left|f_{n}\right|^{2},
$$

so that $\left\{E_{n}\right\},\left\{F_{m}\right\}$ form an orthonormal basis. The operator $z: H \rightarrow H$ is defined by the formula $f(\zeta) \mapsto \zeta f(\zeta)$. With respect to the above basis, the operator $z$ is a rather special weighted bilateral shift. We have

$$
\begin{array}{ll}
z E_{n}=E_{n+1} \quad \text { for } \quad n \geq 0, \\
z F_{n}=r F_{n+1} \quad \text { for } \quad n \leq-2, \\
z F_{-1}=r E_{0}
\end{array}
$$

and

$$
\begin{aligned}
& z^{*} E_{n}=E_{n-1} \quad \text { for } \quad n \geq 1, \\
& z^{*} E_{0}=r F_{-1}, \\
& z^{*} F_{n}=r F_{n-1} \quad \text { for } \quad n \leq-1 .
\end{aligned}
$$

In full analogy with the disk case, the operator $z$ is a form of a noncommutative coordinate for what we call quantum annulus. First we look at the spectrum of $z$.

Theorem 2.4. The norm of $z$ is 1 . The spectrum of $z$ is all of $A_{r}$.

Proof. The formulas above easily imply that $\|z\| \leq 1$, while the action of $z$ on $E_{n}$ shows that it is exactly 1. It is then straightforward to verify that for $\lambda$ inside $A_{r}$ the following is an eigenvector of $z^{*}$ corresponding to the eigenvalue $\lambda$

$$
\phi_{\lambda}=\sum_{n=0}^{\infty} \lambda^{n} E_{n}+\sum_{n=-\infty}^{-1}\left(\frac{\lambda}{r}\right)^{n} F_{n} .
$$

Finally, using the techniques described in Lemma 3.7 below, we can prove that the operator $z-\lambda$ is invertible for $|\lambda|<r$. Put together those statements imply that the spectrum of $z$ is $A_{r}$. 
The operators $z^{*} z$ and $z z^{*}$ are diagonal. We have

$$
\begin{array}{ll}
z z^{*} E_{n}=E_{n} & \text { for } \quad n \geq 1, \\
z z^{*} F_{n}=r^{2} F_{n} & \text { for } \quad n \leq-1, \\
z z^{*} E_{0}=r^{2} E_{0} &
\end{array}
$$

and

$$
\begin{array}{ll}
z^{*} z E_{n}=E_{n} & \text { for } \quad n \geq 1, \\
z^{*} z F_{n}=r^{2} F_{n} & \text { for } \quad n \leq-1, \\
z^{*} z E_{0}=E_{0} . &
\end{array}
$$

Thus the spectrum of those operators is $\sigma\left(z z^{*}\right)=\{1\} \cup\left\{r^{2}\right\}=\sigma\left(z^{*} z\right)$. Also notice that the spectral projections $P_{z^{*} z}(1)$ and $P_{z^{*} z}\left(r^{2}\right)$ of $z^{*} z$ are orthogonal projections onto subspaces of $H$ generated by $E_{n}$ 's and $F_{n}$ 's, respectively. By the continuous functional calculus applied to $z^{*} z$, both projections belong to $C^{*}(z)$, the $C^{*}$-algebra generated by $z$.

Remark 2.5. The above formulas also imply that the commutator $\frac{z^{*} z-z z^{*}}{1-r^{2}}=\frac{\left[z^{*}, z\right]}{1-r^{2}}$ is the orthogonal projection onto the one-dimensional subspace spanned by $E_{0}$, hence a compact operator.

Theorem 2.6. The commutator ideal of $C^{*}(z)$ is the ideal of compact operators.

Proof. By the remark above the commutator ideal of $C^{*}(z)$ is contained in $\mathcal{K}$. Similar to the quantum disk case, the opposite inclusion follows from the easily verifiable fact that the rank one operators $f \mapsto\left\langle f, E_{i}\right\rangle E_{j}, f \mapsto\left\langle f, E_{i}\right\rangle F_{j}, f \mapsto\left\langle f, F_{i}\right\rangle E_{j}, f \mapsto\left\langle f, F_{i}\right\rangle F_{j}$ are in the commutator ideal of $C^{*}(z)$.

Theorem 2.7. The quotient $C^{*}(z) / \mathcal{K}$ is isomorphic to $C\left(S^{1}\right) \oplus C\left(S^{1}\right)$, where $C\left(S^{1}\right)$ is the space of continuous functions on the unit circle. Thus we have a short exact sequence

$$
0 \rightarrow \mathcal{K} \rightarrow C^{*}(z) \rightarrow C\left(S^{1}\right) \oplus C\left(S^{1}\right) \rightarrow 0
$$

Proof. For details we refer to the proof of Theorem 3.15 in the next section. The key step is showing that the infinite-dimensional spectral projections $P_{z^{*} z}(1)$ and $P_{z^{*} z}\left(r^{2}\right)$ are in $C^{*}(z)$. They can be used together with Toeplitz operators on subspaces generated by $E_{n}$ 's and $F_{n}$ 's to construct an isomorphism between $C^{*}(z) / \mathcal{K}$ and $C\left(S^{1}\right) \oplus C\left(S^{1}\right)$ in a similar fashion to the Toeplitz algebra case.

\section{The quantum pair of pants}

Let $0<a<1, a+r_{2}<1, r_{1}+r_{2}<a$. We define the (closed) pair of pants as follows

$$
P P_{\left(a, r_{1}, r_{2}\right)}=\left\{\zeta \in \mathbb{C}:|\zeta| \leq 1,|\zeta| \geq r_{1},|\zeta-a| \geq r_{2}\right\} .
$$

It is clear that every open disk with two nonintersecting circular holes is biholomorphically equivalent to the interior of the one of the above pair of pants. There are some technical advantages to having the holes located as above. To a pair of pants we associate a convenient Hilbert space of holomorphic functions on it and study the operator of multiplication by $\zeta$ on that Hilbert space. This is described more precisely in the following subsection. 


\subsection{Definitions}

It follows from [16] that every holomorphic function on the interior of $P P_{\left(a, r_{1}, r_{2}\right)}$ can be approximated by rational functions with the only singularities at the centers of the smaller circles in $P P_{\left(a, r_{1}, r_{2}\right)}$ or at infinity. In fact we can do a little better.

Proposition 3.1. Every holomorphic function $\varphi(\zeta)$ on the interior of $P P_{\left(a, r_{1}, r_{2}\right)}$ can be written as the following convergent series

$$
\varphi(\zeta)=\sum_{n=0}^{\infty} e_{n} \zeta^{n}+\sum_{n=-\infty}^{-1} f_{n}\left(\frac{\zeta}{r_{1}}\right)^{n}+\sum_{n=-\infty}^{-1} g_{n}\left(\frac{\zeta-a}{r_{2}}\right)^{n} .
$$

Proof. In [3], it was shown that if $\varphi(\zeta)$ is holomorphic on an annulus $\left\{\zeta \in \mathbb{C}: R_{1}<|\zeta-c|<\right.$ $\left.R_{2}\right\}$, then $\varphi(\zeta)=\varphi_{1}(\zeta)+\varphi_{2}(\zeta)$ where $\varphi_{1}(\zeta)$ is holomorphic on $|\zeta-c|>R_{1}$ and $\varphi_{2}(\zeta)$ is holomorphic on $|\zeta-c|<R_{2}$. We apply this theorem twice. Let $\varphi$ be a holomorphic function on the open pair of pants. Consider an annulus $A=\left\{\zeta \in \mathbb{C}:|\zeta-a|>r_{2},|\zeta-c|<r\right\}$ around $a$ with outer radius $r$ and inner radius $r_{2}$ that does not intersect the hole around the origin with radius $r_{1}$ and let $D$ be the disk with center $a$ and radius $r$. Then $\left.\varphi\right|_{A}$ is a holomorphic function and so from [3], $\left.\varphi\right|_{A}=\varphi_{1}+\varphi_{2}$ with $\varphi_{1}$ holomorphic outside the hole centered at $a$ with radius $r_{2}$, and $\varphi_{2}$ holomorphic on $D$. Consequently $\varphi_{1}$ has the following convergent series representation

$$
\varphi_{1}=\sum_{n=-\infty}^{-1} g_{n}\left(\frac{\zeta-a}{r_{2}}\right)^{n} \text {. }
$$

Next consider the function $\varphi-\varphi_{1}$. This function is holomorphic on $P P_{\left(a, r_{1}, r_{2}\right)}$ and, because $\varphi-\varphi_{1}=\varphi_{2}$ on $\mathrm{A}$, it extends to a holomorphic function on $D$. This means that $\varphi-\varphi_{1}$ is holomorphic on the annulus $\left\{\zeta \in \mathbb{C}:|\zeta|>r_{1},|\zeta-c|<1\right\}$, and so by using [3] again, we have $\varphi-\varphi_{1}=\varphi_{3}+\varphi_{4}$ with $\varphi_{3}$ holomorphic in the unit disk $\mathbb{D}$ and $\varphi_{4}$ holomorphic on $\left\{\zeta \in \mathbb{C}:|\zeta|>r_{1}\right\}$. Thus $\varphi_{3}$ and $\varphi_{4}$ have the following convergent series representation

$$
\varphi_{3}=\sum_{n=0}^{\infty} e_{n} \zeta^{n} \quad \text { and } \quad \varphi_{4}=\sum_{n=-\infty}^{-1} f_{n}\left(\frac{\zeta}{r_{1}}\right)^{n} .
$$

Combining these three series representations gives the desired result.

Similar to the annulus case we set

$$
E_{n}=\zeta^{n}, \quad F_{n}=\left(\frac{\zeta}{r_{1}}\right)^{n}, \quad \text { and } \quad G_{n}=\left(\frac{\zeta-a}{r_{2}}\right)^{n}
$$

The Hilbert space $H$ that we will use is defined as

$$
H=\left\{\varphi(\zeta)=\sum_{n=0}^{\infty} e_{n} E_{n}+\sum_{n=-\infty}^{-1}\left(f_{n} F_{n}+g_{n} G_{n}\right):\|\varphi\|<\infty\right\},
$$

where

$$
\|\varphi\|^{2}=\sum_{n=0}^{\infty}\left|e_{n}\right|^{2}+\sum_{n=-\infty}^{-1}\left(\left|f_{n}\right|^{2}+\left|g_{n}\right|^{2}\right)
$$

The advantage of working with the above Hilbert space of holomorphic functions on $P P_{\left(a, r_{1}, r_{2}\right)}$ is that there is a distinguished orthonormal basis in it, namely the basis consisting of $\left\{E_{n}\right\},\left\{F_{m}\right\}$, $\left\{G_{k}\right\}$.

The object of study in this section is the operator $z: H \rightarrow H$ given by $z \varphi(\zeta)=M_{\zeta} \varphi(\zeta)=$ $\zeta \varphi(\zeta)$, i.e., the multiplication operator by $\zeta$. Straightforward calculations yields the following formulas. 
Lemma 3.2. The operators $z$ and $z^{*}$ act on the basis elements in the following way

$$
\begin{array}{ll}
z E_{n}=E_{n+1} & \text { for } \quad n \geq 0, \\
z F_{n}=r_{1} F_{n+1} & \text { for } \quad n \leq-2, \\
z F_{-1}=r_{1} E_{0}, & \\
z G_{n}=r_{2} G_{n+1}+a G_{n} & \text { for } \quad n \leq-2, \\
z G_{-1}=r_{2} E_{0}+a G_{-1} &
\end{array}
$$

and

$$
\begin{array}{ll}
z^{*} E_{n}=E_{n-1} & \text { for } \quad n \geq 1, \\
z^{*} E_{0}=r_{1} F_{-1}+r_{2} G_{-1}, & \\
z^{*} F_{n}=r_{1} F_{n-1} & \text { for } \quad n \leq-1, \\
z^{*} G_{n}=r_{2} G_{n-1}+a G_{n} & \text { for } n \leq-1 .
\end{array}
$$

Lemma 3.3. The operators $z$ and $z^{*}$ shift the coefficients of $\varphi(\zeta)$ in the series decomposition defined in equation (3.1) in the following way

$$
z \varphi=\sum_{n=0}^{\infty} \tilde{e}_{n} E_{n}+\sum_{n=-\infty}^{-1}\left(\tilde{f}_{n} F_{n}+\tilde{g}_{n} G_{n}\right) \quad \text { and } \quad z^{*} \varphi=\sum_{n=0}^{\infty} e_{n}^{\prime} E_{n}+\sum_{n=-\infty}^{-1}\left(f_{n}^{\prime} F_{n}+g_{n}^{\prime} G_{n}\right),
$$

where

$$
\begin{array}{lll}
\tilde{e}_{n}=e_{n-1} & \text { for } & n \geq 1, \\
\tilde{e}_{0}=r_{1} f_{-1}+r_{2} g_{-1}, & & \\
\tilde{f}_{n}=r_{1} f_{n-1} & \text { for } & n \leq-1, \\
\tilde{g}_{n}=r_{2} g_{n-1}+a g_{n} & \text { for } \quad n \leq-1
\end{array}
$$

and

$$
\begin{array}{ll}
e_{n}^{\prime}=e_{n+1} & \text { for } \quad n \geq 0, \\
f_{n}^{\prime}=r_{1} f_{n+1} & \text { for } \quad n \leq-2, \\
f_{-1}^{\prime}=r_{1} e_{0}, & \\
g_{n}^{\prime}=r_{2} g_{n+1}+a g_{n} & \text { for } n \leq-2, \\
g_{-1}^{\prime}=r_{2} e_{0}+a g_{-1} . &
\end{array}
$$

We can now define the quantum pair of pants.

Definition 3.4. The quantum pair of pants, denoted $Q P P_{\left(a, r_{1}, r_{2}\right)}$, is defined to be the $C^{*}$ algebra generated by the operator $z$, i.e., $Q P P_{\left(a, r_{1}, r_{2}\right)}=C^{*}(z)$.

\subsection{The spectrum of $z$}

In this subsection we study the spectrum of $z$, starting with a calculation of the norm of $z$.

Proposition 3.5. With the above notation, we have: $\|z\|=1$.

Proof. Using the series representation of $\varphi(\zeta)$ in formula (3.1) above, and the coefficients of Lemma 3.3 we compute $\|z \varphi\|^{2}$ :

$$
\|z \varphi\|^{2}=\sum_{n=1}^{\infty}\left|e_{n-1}\right|^{2}+\left|r_{1} f_{-1}+r_{2} g_{-1}\right|^{2}+r_{1}^{2} \sum_{n=-\infty}^{-1}\left|f_{n-1}\right|^{2}+\sum_{n=-\infty}^{-1}\left|r_{2} g_{n-1}+a g_{n}\right|^{2} .
$$


Using the triangle inequality and the fact that $a>r_{1}$ we obtain

$$
\|z \varphi\|^{2} \leq \sum_{n=0}^{\infty}\left|e_{n}\right|^{2}+\left(a\left|f_{-1}\right|+r_{2}\left|g_{-1}\right|\right)^{2}+r_{1}^{2} \sum_{n=-\infty}^{-1}\left|f_{n-1}\right|^{2}+\sum_{n=-\infty}^{-1}\left(r_{2}\left|g_{n-1}\right|+a\left|g_{n}\right|\right)^{2} .
$$

Notice that by denoting $g_{0}:=f_{-1}$ we can write

$$
\begin{aligned}
& \left(a\left|f_{-1}\right|+r_{2}\left|g_{-1}\right|\right)^{2}+\sum_{n=-\infty}^{-1}\left(r_{2}\left|g_{n-1}\right|+a\left|g_{n}\right|\right)^{2}=\sum_{n=-\infty}^{0}\left(r_{2}\left|g_{n-1}\right|+a\left|g_{n}\right|\right)^{2} \\
& \quad=r_{2}^{2} \sum_{n=-\infty}^{0}\left|g_{n-1}\right|^{2}+a^{2} \sum_{n=-\infty}^{0}\left|g_{n}\right|^{2}+2 a r_{2} \sum_{n=-\infty}^{0}\left|g_{n-1}\right|\left|g_{n}\right| .
\end{aligned}
$$

The Cauchy-Schwartz inequality implies

$$
\begin{aligned}
& \sum_{n=-\infty}^{0}\left(r_{2}\left|g_{n-1}\right|+a\left|g_{n}\right|\right)^{2} \leq r_{2}^{2} \sum_{n=-\infty}^{0}\left|g_{n-1}\right|^{2}+a^{2} \sum_{n=-\infty}^{0}\left|g_{n}\right|^{2} \\
& \quad+2 a r_{2}\left(\sum_{n=-\infty}^{0}\left|g_{n-1}\right|^{2}\right)^{1 / 2}\left(\sum_{n=-\infty}^{0}\left|g_{n}\right|^{2}\right)^{1 / 2} \\
& \leq\left(r_{2}^{2}+a^{2}+2 a r_{2}\right) \sum_{n=-\infty}^{0}\left|g_{n}\right|^{2}=\left(r_{2}+a\right)^{2}\left(\left|f_{-1}\right|^{2}+\sum_{n=-\infty}^{-1}\left|g_{n}\right|^{2}\right) .
\end{aligned}
$$

Using the fact that $r_{1}, r_{2}+a<1$ in the above computations we see that

$$
\begin{aligned}
\|z \varphi\|^{2} & \leq \sum_{n=0}^{\infty}\left|e_{n}\right|^{2}+r_{1}^{2} \sum_{n=\infty}^{-1}\left|f_{n-1}\right|^{2}+\left(r_{2}+a\right)^{2}\left|f_{-1}\right|^{2}+\left(r_{2}+a\right)^{2} \sum_{n=-\infty}^{-1}\left|g_{n}\right|^{2} \\
& \leq \sum_{n=0}^{\infty}\left|e_{n}\right|^{2}+\sum_{n=\infty}^{-1}\left|f_{n}\right|^{2}+\sum_{n=-\infty}^{-1}\left|g_{n}\right|^{2}=\|\varphi\|^{2}
\end{aligned}
$$

showing that $\|z\| \leq 1$. On the other hand, $\left\|z E_{1}\right\|=\left\|E_{2}\right\|=\left\|E_{1}\right\|$. Thus $\|z\|=1$.

Next we compute the spectrum of $z$. In estimating the norms of resolvents of $z$ we use the following well known result.

Lemma 3.6 (Schur-Young inequality). Let $T: L^{2}(Y) \longrightarrow L^{2}(X)$ be an integral operator

$$
T f(x)=\int K(x, y) f(y) d y
$$

Then one has

$$
\|T\|^{2} \leq\left(\sup _{x \in X} \int_{Y}|K(x, y)| d y\right)\left(\sup _{y \in Y} \int_{X}|K(x, y)| d x\right) .
$$

The details of the lemma and its proof can be found in [6].

Lemma 3.7. The operator $z-\lambda$ has a bounded inverse for $|\lambda|<r_{1},|\lambda-a|<r_{2}$, and $|\lambda|>1$. 
Proof. Let

$$
\varphi(\zeta)=\sum_{n=0}^{\infty} e_{n} E_{n}+\sum_{n=-\infty}^{-1}\left(f_{n} F_{n}+g_{n} G_{n}\right)
$$

and

$$
\tilde{\varphi}(\zeta)=\sum_{n=0}^{\infty} \tilde{e}_{n} E_{n}+\sum_{n=-\infty}^{-1}\left(\tilde{f}_{n} F_{n}+\tilde{g}_{n} G_{n}\right) .
$$

Consider the equation $(z-\lambda) \varphi(\zeta)=\tilde{\varphi}(\zeta)$. Using the above decompositions and Lemma 3.3 we obtain the following system of equations

$$
\begin{array}{ll}
r_{1} f_{-1}+r_{2} g_{-1}-\lambda e_{0}=\tilde{e}_{0}, & \\
e_{n-1}-\lambda e_{n}=\tilde{e}_{n} & \text { for } \quad n \geq 1, \\
r_{1} f_{n-1}-\lambda f_{n}=\tilde{f}_{n} & \text { for } \quad n \leq-1, \\
r_{2} g_{n-1}+a g_{n}-\lambda g_{n}=\tilde{g}_{n} & \text { for } \quad n \leq-1 .
\end{array}
$$

By Proposition 3.5, $\|z\|=1$ and if $|\lambda|>1=\|z\|$ then by general functional analysis we know that $(z-\lambda)^{-1}$ is a bounded, invertible operator.

Next we consider three cases: the first case is for $0<|\lambda|<r_{1}$, the second case is for $|\lambda-a|<r_{2}$, and the last case is for $\lambda=0$.

If $0<|\lambda|<r_{1}<1$, then $|\lambda-a|>r_{2}$. We can solve the system of equations (3.2) recursively. Rewriting the last equation and multiplying by $\left((\lambda-a) / r_{2}\right)^{n-1}$ yields

$$
\left(\frac{\lambda-a}{r_{2}}\right)^{n-1} g_{n-1}-\left(\frac{\lambda-a}{r_{2}}\right)^{n} g_{n}=\left(\frac{\lambda-a}{r_{2}}\right)^{n-1} \frac{1}{r_{2}} \tilde{g}_{n} .
$$

Letting $h_{n}=\left((\lambda-a) / r_{2}\right)^{n} g_{n}$, we get

$$
h_{n-1}-h_{n}=\left(\frac{\lambda-a}{r_{2}}\right)^{n-1} \frac{1}{r_{2}} \tilde{g}_{n} \text {. }
$$

The requirement for a square summable solution forces $h_{n}=-\sum_{j=-\infty}^{n}\left((\lambda-a) / r_{2}\right)^{j-1} \tilde{g}_{j} / r_{2}$ and hence for $n \leq-1$ we obtain

$$
g_{n}=-\frac{1}{r_{2}} \sum_{j=-\infty}^{n}\left(\frac{\lambda-a}{r_{2}}\right)^{j-n-1} \tilde{g}_{j} .
$$

Similar calculations show that

$$
e_{n}=\sum_{j=n+1}^{\infty} \lambda^{j-n-1} \tilde{e}_{j} \quad \text { and } \quad f_{n}=\left(\frac{\lambda}{r_{1}}\right)^{-n-1} f_{-1}+\frac{1}{r_{1}} \sum_{j=n+1}^{-1}\left(\frac{\lambda}{r_{1}}\right)^{j-n-1} \tilde{f}_{j}
$$

for $n \geq 0$ and $n \leq-2$ respectively. These formulas along with the first equation in system (3.2) give

$$
f_{-1}=\frac{1}{r_{1}}\left(\sum_{j=0}^{\infty} \lambda^{j} \tilde{e}_{j}+\sum_{j=-\infty}^{-1}\left(\frac{\lambda-a}{r_{2}}\right)^{j} \tilde{g}_{j}\right) .
$$


We introduce some notation; first notice that we have a natural decomposition, $H \cong \ell^{2}\left(\mathbb{Z}_{\geq 0}\right) \oplus$ $\ell^{2}\left(\mathbb{Z}_{<0}\right) \oplus \ell^{2}\left(\mathbb{Z}_{<0}\right)$ given in the following way: for $\varphi \in H$ write $\varphi=e+f+g$ where $e=\sum_{n>0} e_{n} E_{n}$, $f=\sum_{n \leq-1} f_{n} F_{n}$, and $g=\sum_{n \leq-1} g_{n} G_{n}$. Using this notation we see that $\|\varphi\|^{2}=\|e\|^{2}+\|f\|^{2}+\|g\|^{2}$. Define the characteristic $\chi(t)=1$ for $0 \leq t \leq 1$ and zero otherwise, then we can define seven different integral operators

$$
\begin{aligned}
& T_{1} e=\sum_{n=0}^{\infty} \sum_{j=0}^{\infty} \lambda^{j-n-1} \chi\left(\frac{n+1}{j}\right) e_{j} E_{n}: \ell^{2}\left(\mathbb{Z}_{\geq 0}\right) \rightarrow \ell^{2}\left(\mathbb{Z}_{\geq 0}\right), \\
& T_{2}(e, g)=\sum_{n=-\infty}^{-1} \frac{1}{r_{1}}\left(\frac{\lambda}{r_{1}}\right)^{-n-1}\left(\sum_{j=0}^{\infty} \lambda^{j} e_{j}+\sum_{j=-\infty}^{-1}\left(\frac{\lambda-a}{r_{2}}\right)^{j} g_{j}\right) F_{n}: \\
& \ell^{2}\left(\mathbb{Z}_{\geq 0}\right) \oplus \ell^{2}\left(\mathbb{Z}_{<0}\right) \rightarrow \ell^{2}\left(\mathbb{Z}_{<0}\right) \\
& T_{3} f=\sum_{n=-\infty}^{-1} \frac{1}{r_{1}} \sum_{j=-\infty}^{-1}\left(\frac{\lambda}{r_{1}}\right)^{j-n-1} \chi\left(\frac{n+1}{j}\right) f_{j} F_{n}: \ell^{2}\left(\mathbb{Z}_{<0}\right) \rightarrow \ell^{2}\left(\mathbb{Z}_{<0}\right), \\
& T_{4} f=\sum_{n=-\infty}^{-1} \frac{1}{r_{1}} \sum_{j=-\infty}^{-1}\left(\frac{\lambda}{r_{1}}\right)^{j-n-1} \chi\left(\frac{j}{n}\right) f_{j} F_{n}: \ell^{2}\left(\mathbb{Z}_{<0}\right) \rightarrow \ell^{2}\left(\mathbb{Z}_{<0}\right), \\
& T_{5}(e, f)=\sum_{n=-\infty}^{-1} \frac{1}{r_{2}}\left(\frac{\lambda-a}{r_{2}}\right)^{-n-1}\left(\sum_{j=0}^{\infty} \lambda^{j} e_{j}+\sum_{j=-\infty}^{-1}\left(\frac{\lambda}{r_{1}}\right)^{j} f_{j}\right) G_{n}: \\
& T_{6} g=\sum_{n=-\infty}^{-1} \frac{1}{r_{2}} \sum_{j=-\infty}\left(\frac{\lambda-a}{r_{2}}\right)^{j-n-1} \chi\left(\frac{n+1}{j}\right) \ell_{j} G_{n}: \ell^{2}\left(\mathbb{Z}_{<0}\right) \rightarrow \ell^{2}\left(\mathbb{Z}_{<0}\right), \\
& T_{7} g=\sum_{n=-\infty}^{-1} \frac{1}{r_{2}} \sum_{j=-\infty}^{-1}\left(\frac{\lambda-a}{r_{2}}\right)^{j-n-1} \chi\left(\frac{j}{n}\right) g_{j} G_{n}: \ell^{2}\left(\mathbb{Z}_{<0}\right) \rightarrow \ell^{2}\left(\mathbb{Z}_{<0}\right) .
\end{aligned}
$$

The operators from formula (3.3) can be used to represent $(z-\lambda)^{-1} \tilde{\varphi}$, for $\tilde{\varphi}=\tilde{e}+\tilde{f}+\tilde{g}$ where $\tilde{e}=\sum_{n \geq 0} \tilde{e}_{n} E_{n}, \tilde{f}=\sum_{n \leq-1} \tilde{f}_{n} F_{n}$ and $\tilde{g}=\sum_{n \leq-1} \tilde{g}_{n} G_{n}$, in the following way

$$
(z-\lambda)^{-1} \tilde{\varphi}=T_{1} \tilde{e}+T_{2}(\tilde{e}, \tilde{g})+T_{3} \tilde{f}-T_{7} \tilde{g}
$$

Next we estimate the norm of $(z-\lambda)^{-1}$. We use Lemma 3.6 to estimate the norms of the operators $T_{1}, T_{3}$ and $T_{7}$ and we directly estimate $\left\|T_{2} \tilde{f}\right\|$. The first estimate is

$$
\begin{aligned}
\left\|T_{1}\right\|^{2} & \leq\left(\sup _{n \geq 0}|\lambda|^{-n-1} \sum_{j=n+1}^{\infty}|\lambda|^{j}\right)\left(\sup _{j \geq 1}|\lambda|^{j-1} \sum_{n=0}^{j-1}|\lambda|^{-n}\right) \\
& =\frac{1}{(1-|\lambda|)}\left(\sup _{j \geq 1} \frac{1-|\lambda|^{j}}{1-|\lambda|}\right)=\frac{1}{(1-|\lambda|)^{2}},
\end{aligned}
$$

where we have used the fact that $|\lambda|<1$. Similarly, we have

$$
\left\|T_{3}\right\|^{2} \leq \frac{1}{r_{1}^{2}}\left(\sup _{n \leq-2}\left(\frac{|\lambda|}{r_{1}}\right)^{-n-1} \sum_{j=n+1}^{-1}\left(\frac{|\lambda|}{r_{1}}\right)^{j}\right)\left(\sup _{j \leq-1}\left(\frac{|\lambda|}{r_{1}}\right)^{j-1} \sum_{n=-\infty}^{j-1}\left(\frac{|\lambda|}{r_{1}}\right)^{-n}\right)
$$




$$
\leq \frac{1}{r_{1}^{2}\left(1-\frac{|\lambda|}{r_{1}}\right)^{2}}\left(\sup _{n \leq-2} 1-\left(\frac{|\lambda|}{r_{1}}\right)^{-n-1}\right)
$$

Since $\frac{|\lambda|}{r_{1}}<1$, it follows that $\left\|T_{3}\right\|^{2} \leq \frac{1}{r_{1}^{2}\left(1-\frac{|\lambda|}{r_{1}}\right)^{2}}$.

Next,

$$
\begin{aligned}
\left\|T_{7}\right\|^{2} & \leq \frac{1}{r_{2}^{2}}\left(\sup _{n \leq-1}\left|\frac{\lambda-a}{r_{2}}\right|^{-n-1} \sum_{j=-\infty}^{n}\left|\frac{\lambda-a}{r_{2}}\right|^{j}\right)\left(\sup _{j \leq-1}\left|\frac{\lambda-a}{r_{2}}\right|^{j-1} \sum_{n=j}^{-1}\left|\frac{\lambda-a}{r_{2}}\right|^{-n}\right) \\
& \leq \frac{1}{r_{2}^{2}} \frac{\left(|\lambda-a| / r_{2}\right)^{-2}}{\left(1-\left(|\lambda-a| / r_{2}\right)^{-1}\right)^{2}}\left(\sup _{j \leq-1} 1-\left(\frac{|\lambda-a|}{r_{2}}\right)^{j}\right) .
\end{aligned}
$$

Because $\frac{|\lambda-a|}{r_{2}}>1$, we have

$$
\left\|T_{7}\right\|^{2} \leq \frac{1}{r_{2}^{2}} \frac{\left(|\lambda-a| / r_{2}\right)^{-2}}{\left(1-\left(|\lambda-a| / r_{2}\right)^{-1}\right)^{2}}=\frac{1}{r_{1}^{2}\left(\frac{|\lambda-a|}{r_{2}}-1\right)^{2}} .
$$

The operator $T_{2}$ is a rank one operator and the norm $T_{2} \tilde{f}$ can be estimated directly, using the Cauchy-Schwartz inequality

$$
\begin{aligned}
\left\|T_{2}(\tilde{e}, \tilde{g})\right\|^{2} & =\frac{1}{r_{1}^{2}} \sum_{n=-\infty}^{-1}\left(\frac{|\lambda|}{r_{1}}\right)^{-2 n-2}\left|\sum_{j=0}^{\infty} \lambda^{j} \tilde{e}_{j}+\sum_{j=-\infty}^{-1}\left(\frac{\lambda-a}{r_{2}}\right)^{j} \tilde{g}_{j}\right|^{2} \\
& \leq \frac{1}{r_{1}^{2}\left(1-\left(|\lambda| / r_{1}\right)^{2}\right)}\left(\frac{1}{1-|\lambda|^{2}}+\frac{1}{\left(|\lambda-a| / r_{2}\right)^{2}-1}\right)\left(\|\tilde{e}\|^{2}+\|\tilde{g}\|^{2}\right) .
\end{aligned}
$$

This shows that $(z-\lambda)^{-1}$ is bounded for $0<|\lambda|<r_{1}$.

The second case is $|\lambda-a|<r_{2}$. This implies that $r_{1}<|\lambda|<1$. Under these constraints we solve system (3.2) using the same methods as those for the first case to obtain

$$
\begin{aligned}
& e_{n}=\sum_{j=n+1}^{\infty} \lambda^{j-n-1} \tilde{e}_{j} \quad \text { for } \quad n \geq 0, \\
& f_{n}=-\frac{1}{r_{1}} \sum_{j=-\infty}^{n}\left(\frac{\lambda}{r_{1}}\right)^{j-n-1} \tilde{f}_{j} \quad \text { for } \quad n \leq-1, \\
& g_{n}=-\left(\frac{\lambda-a}{r_{2}}\right)^{-n-1} g_{-1}+\frac{1}{r_{2}} \sum_{j=n+1}^{-1}\left(\frac{\lambda-a}{r_{2}}\right)^{j-n-1} \tilde{g}_{j} \quad \text { for } \quad n \leq-2 .
\end{aligned}
$$

Then the first equation of system (3.2) gives

$$
g_{-1}=\frac{1}{r_{2}}\left(\sum_{j=0}^{\infty} \lambda^{j} \tilde{e}_{j}+\sum_{j=-\infty}^{-1}\left(\frac{\lambda}{r_{1}}\right)^{j} \tilde{f}_{j}\right) .
$$

Similar to the first case we can express $(z-\lambda)^{-1}$ using the operators defined in formula (3.3) to get

$$
(z-\lambda)^{-1} \tilde{\varphi}=T_{1} \tilde{e}-T_{4} \tilde{f}+T_{5}(\tilde{e}, \tilde{f})+T_{6} \tilde{g} .
$$


We omit the repetitive details of estimates of $T_{4}, T_{5}$, and $T_{6}$ norms. They imply that $(z-\lambda)^{-1}$ is bounded for $|\lambda-a|<r_{2}$.

The last case is when $\lambda=0$. Solving system (3.2) we obtain

$$
\begin{aligned}
& e_{n}=\tilde{e}_{n+1} \quad \text { for } \quad n \geq 0, \\
& f_{n}=\frac{1}{r_{1}} \tilde{f}_{n+1} \quad \text { for } \quad n \leq-2, \\
& g_{n}=-\frac{1}{r_{2}} \sum_{j=-\infty}^{n}\left(-\frac{a}{r_{2}}\right)^{j-n-1} \tilde{g}_{j} \quad \text { for } \quad n \leq-1 .
\end{aligned}
$$

Using the first equation of system (3.2) we compute $f_{-1}$

$$
f_{-1}=\frac{1}{r_{1}}\left(\tilde{e}_{0}+\sum_{j=-\infty}^{-1}\left(-\frac{a}{r_{2}}\right)^{j} \tilde{g}_{j}\right) .
$$

As before the norm estimates hinge on convergent geometric series. This completes the proof.

Theorem 3.8. The spectrum of $z$ is the regular pair of pants, i.e., $\sigma(z)=P P_{\left(a, r_{1}, r_{2}\right)}$.

Proof. By Proposition 3.5, $\sigma(z) \subset \mathbb{D}$. Let

$$
\varphi_{\lambda}(\zeta)=\sum_{n=0}^{\infty} \lambda^{n} E_{n}+\sum_{n=-\infty}^{-1}\left(\frac{\lambda}{r_{1}}\right)^{n} F_{n}+\sum_{n=-\infty}^{-1}\left(\frac{\lambda-a}{r_{2}}\right)^{n} G_{n} .
$$

It is easy to see that for any $\lambda$ in the interior of $P P_{\left(a, r_{1}, r_{2}\right)}, \varphi_{\lambda} \in H$ and that $\lambda$ is an eigenvalue with $\varphi_{\lambda}$ as the associated eigenfunction for $z^{*}$. Therefore, $P P_{\left(a, r_{1}, r_{2}\right)} \subset \sigma(z)$. From Lemma 3.7 the operator $z-\lambda$ has a bounded inverse whenever $|\lambda|<r_{1}$ or $|\lambda-a|<r_{2}$. Hence the resolvent set is contained in the holes within the unit disk or outside the unit disk, and so $\sigma(z) \subset P P_{\left(a, r_{1}, r_{2}\right)}$.

In view of the above theorem we can think of the operator $z$ as a form of a noncommutative complex coordinate for what we call quantum pair of pants.

\subsection{Structure of $C^{*}(z)$}

Next we study the commutator ideal of $C^{*}(z)$. A straightforward computation gives the following formulas.

Lemma 3.9. The commutator of $z^{*}$ and $z$ act on the basis elements in the following way: $\left[z^{*}, z\right] E_{n}=0$ for $n \geq 1,\left[z^{*}, z\right] F_{n}=0$ for $n \leq-2,\left[z^{*}, z\right] G_{n}=0$ for $n \leq-2$. Moreover on the initial elements we get:

$$
\begin{aligned}
& {\left[z^{*}, z\right] E_{0}=\left(1-r_{1}^{2}-r_{2}^{2}\right) E_{0}-a r_{2} G_{-1},} \\
& {\left[z^{*}, z\right] F_{-1}=r_{1} r_{2} G_{-1},} \\
& {\left[z^{*}, z\right] G_{-1}=r_{1} r_{2} F_{-1}-a r_{2} E_{0} .}
\end{aligned}
$$

Let $\mathcal{I}$ be the ideal generated by $\left[z^{*}, z\right]$. It is easy to see that $\mathcal{I}$ is in fact the commutator ideal of $C^{*}(z)$ because that algebra is singly generated.

Theorem 3.10. The commutator ideal $\mathcal{I}$ of $C^{*}(z)$ is the $C^{*}$-algebra $\mathcal{K}$ of compact operators in $H$. 
Proof. From Lemma 3.9 it is clear that the commutator $\left[z^{*}, z\right]$ is finite-rank and hence compact. Thus, $\mathcal{I} \subset \mathcal{K}$. On the other hand, to show that $\mathcal{K} \subset \mathcal{I}$ we will use the following step by step method building up to the conclusion that a large collection of rank one operators belong to $\mathcal{I}$ and that the compact operators are exactly the norm limit of those.

Step 1. First we show that $P=$ orthogonal projection onto $\operatorname{span}\left\{E_{0}, F_{-1}, G_{-1}\right\}$ belongs to the commutator ideal. Notice that the (self-adjoint) operator $\left[z^{*}, z\right]$ acting on $\operatorname{span}\left\{E_{0}, F_{-1}, G_{-1}\right\}$ has the following matrix representation in the basis $\left\{E_{0}, F_{-1}, G_{-1}\right\}$ :

$$
A=\left(\begin{array}{ccc}
1-r_{1}^{2}-r_{2}^{2} & 0 & -a r_{2} \\
0 & 0 & r_{1} r_{2} \\
-a r_{2} & r_{1} r_{2} & 0
\end{array}\right) .
$$

This matrix has rank equal to 3 and the following characteristic polynomial

$$
p_{A}(\lambda)=\lambda^{3}-\left(1-r_{1}^{2}-r_{2}^{2}\right) \lambda^{2}-\left(a^{2} r_{2}^{2}+r_{1}^{2} r_{2}^{2}\right) \lambda+\left(1-r_{1}^{2}-r_{2}^{2}\right) r_{1}^{2} r_{2}^{2} .
$$

Since $0<r_{1}, r_{2}<1$ it is clear that zero is not an eigenvalue of $A$. If $\lambda_{i}, i=1,2,3$ are the roots of $p_{A}(\lambda)$ then by functional calculus there exists a continuous function $f: \mathbb{R} \rightarrow \mathbb{R}$ such that $f(0)=0, f\left(\lambda_{i}\right)=1$ so that $f\left(\left[z^{*}, z\right]\right)=P$. Consequently $P \in \mathcal{I} \subset C^{*}(z)$.

Step 2. The next step is showing that $P_{E_{1}}=$ orthogonal projection onto span of $\left\{E_{1}\right\}$ belongs to $\mathcal{I}$. We first observe that the operator $z P z^{*}$ acts on the basis elements in the following way

$$
z p z^{*} B= \begin{cases}\left(r_{1}^{2}+r_{2}^{2}\right) E_{0}+a r_{2} G_{-1} & \text { if } B=E_{0}, \\ E_{1} & \text { if } B=E_{1}, \\ a r_{2} E_{0}+a^{2} G_{-1} & \text { if } B=G_{-1}, \\ 0 & \text { otherwise }\end{cases}
$$

Thus, the operator $z P z^{*}$ on $\operatorname{span}\left\{E_{0}, G_{-1}\right\}$ is self-adjoint and has the following matrix representation in the basis $\left\{E_{0}, G_{-1}\right\}$ :

$$
C=\left(\begin{array}{cc}
r_{1}^{2}+r_{2}^{2} & a r_{2} \\
a r_{2} & a^{2}
\end{array}\right)
$$

The characteristic polynomial for $C$ is $p_{C}(\lambda)=\lambda^{2}-\left(r_{1}^{2}+r_{2}^{2}+a^{2}\right) \lambda+a^{2} r_{1}^{2}$. First we need to show that $\lambda=0,1$ are not roots of $p_{C}(\lambda)$. Clearly $p_{C}(0) \neq 0$. Suppose $p_{C}(1)=0$. Then solving the equation for $r_{2}$ we obtain, $r_{2}^{2}=\left(1-a^{2}\right)\left(1-r_{1}^{2}\right)$. Since $r_{2}<1-a$ and $r_{2}<1-r_{1}$ we see that

$$
\left(1-a^{2}\right)\left(1-r_{1}^{2}\right)<(1-a)\left(1-r_{1}\right)
$$

implying $(1+a)\left(1+r_{1}\right)<1$ which is clearly a contradiction since $a, r_{1}>0$. Thus, $p_{C}(1) \neq 0$.

Now we look at the discriminant $\Delta$ of $p_{C}(\lambda)$ :

$$
\Delta=\left(r_{1}^{2}+r_{2}^{2}+a^{2}\right)^{2}-4 a^{2} r_{1}^{2} .
$$

If $\Delta=0$ this would imply that $a=r_{1}$ and $r_{2}=0$, which is a contradiction. Hence $C$ has two distinct eigenvalues $\lambda_{1}$ and $\lambda_{2}$. Thus, once again by functional calculus there exists a continuous real valued function $f$ such that $f(0)=f\left(\lambda_{1}\right)=f\left(\lambda_{2}\right)=0$ and $f(1)=1$. Consequently, applying $f$ to $z P z^{*}$ we get that, $f\left(z P z^{*}\right)=p_{E_{1}} \in \mathcal{I}$.

Step 3. By similar functional calculus argument as above we also see that $P_{E_{0}, G_{-1}}$, the orthogonal projection onto $\operatorname{span}\left\{E_{0}, G_{-1}\right\}$, belongs to $\mathcal{I}$. Consequently, if $P_{F_{-1}}=$ orthogonal projection onto span of $\left\{F_{-1}\right\}$ then clearly, $P_{F_{-1}}=P-P_{E_{0}, G_{-1}} \in \mathcal{I}$. 
Step 4. We will show that $P_{E_{n}}=$ orthogonal projection onto $\operatorname{span}\left\{E_{n}\right\}$ belongs to $\mathcal{I}$ for $n=1,2, \ldots$ To this end, we compute the action of $z^{n-1} P_{E_{1}}\left(z^{*}\right)^{n-1}$ on the basis elements

$$
z^{n-1} P_{E_{1}}\left(z^{*}\right)^{n-1} B= \begin{cases}E_{n} & \text { if } B=E_{n} \\ 0 & \text { otherwise }\end{cases}
$$

Therefore, $z^{n-1} P_{E_{1}}\left(z^{*}\right)^{n-1}=P_{E_{n}} \in \mathcal{I}$ for $n \geq 1$.

Step 5. Now consider the action of $\left(z^{*}\right)^{n} P_{F_{-1}} z^{n}, n \geq 1$ on basis elements

$$
\left(z^{*}\right)^{n} P_{F_{-1}} z^{n} B= \begin{cases}r_{1}^{2 n} F_{-n-1} & \text { if } B=F_{-n-1}, \\ 0 & \text { otherwise }\end{cases}
$$

Thus, $r_{1}^{2 n}\left(z^{*}\right)^{-n} P_{F_{-1}} z^{-n}=P_{F_{n}}$, the projection onto $F_{n}$, for $n \leq-1$ and hence the projections $P_{F_{n}}$ belong to $\mathcal{I}$.

Step 6. Since $z^{*} P_{E_{1}} z E_{0}=E_{0}$ and zero elsewhere, it is clear that $z^{*} P_{E_{1}} z=P_{E_{0}} \in \mathcal{I}$. Hence $P_{G_{-1}}=P-P_{E_{0}}-P_{F_{-1}}$ also belongs to $\mathcal{I}$.

Step 7. It remains to show that for $n \leq-2$ the orthogonal projection $P_{G_{n}}$ onto $G_{n}$ belongs to $\mathcal{I}$. We consider the action of $z^{*} P_{G_{-1}} z$ on basis elements

$$
z^{*} P_{G_{-1}} z B= \begin{cases}a^{2} G_{-1}+a r_{2} G_{-2} & \text { if } B=G_{-1} \\ a r_{2} G_{-1}+r_{2}^{2} G_{-2} & \text { if } B=G_{-2} \\ 0 & \text { otherwise }\end{cases}
$$

Thus it has the following matrix representation relative to the basis $\left\{G_{-1}, G_{-2}\right\}$ :

$$
D=\left(\begin{array}{cc}
a^{2} & a r_{2} \\
a r_{2} & r_{2}^{2}
\end{array}\right)
$$

The matrix $D$ has eigenvalues $\lambda_{1}=0$ and $\lambda_{2}=a^{2}+r_{2}^{2}$ with $v=a G_{-1}+r_{2} G_{-2}$ being the eigenvector corresponding to $\lambda_{2}$. Thus $P_{v}$, the one-dimensional orthogonal projection onto $v /\|v\|$, belongs to $\mathcal{I}$. Since $P_{G_{-1}}$ and $P_{v}$ are not mutually orthogonal, simple matrix algebra shows that the set $\left\{I, P_{G_{-1}}, P_{v}, P_{G_{-1}} P_{v}\right\}$, where $I$ is the $2 \times 2$ identity matrix, generates the set of all $2 \times 2$ matrices. Consequently, $P_{G_{-2}}$ can be written as a linear combination of these four projections, making it clear that $P_{G_{-2}} \in \mathcal{I}$.

Finally we use induction on $n$ and follow a similar argument as above to show that $P_{G_{n}} \in \mathcal{I}$ for $n=-2,-3,-4, \ldots$.

Step 8. Next we proceed to show that the one-dimensional operators $P_{B_{i}, B_{j}}(x)=\left\langle B_{i}, x\right\rangle B_{j}$, where $B_{i}, B_{j}$ are basis elements, i.e., elements of the set $\left\{E_{n}, F_{k}, G_{k}: n \geq 0, k \leq-1\right\}$, also belong to $\mathcal{I}$.

Since $z^{m} P_{E_{n}} E_{n}=E_{n+m}$ we see that $P_{E_{n}, E_{n+m}}=z^{m} P_{E_{n}}$ for every $n, m \geq 0$. Similarly we observe that $P_{E_{n}, E_{n-m}}=\left(z^{*}\right)^{m} P_{E_{n}}$ for $m \leq n$. Together, this proves that all operators $P_{E_{n}, E_{k}} \in \mathcal{I}$ for any $n, k \geq 0$.

Next, we observe that

$$
z^{m} P_{F_{n}}=\left\{\begin{array}{lll}
r_{1}^{m} F_{n+m} & \text { if } & n+m<0, \\
r_{1}^{-n} E_{n+m} & \text { if } & n+m \geq 0 .
\end{array}\right.
$$

Moreover, $\left(z^{*}\right)^{m} P_{F_{n}}=r_{1}^{m} F_{n-m}$ for all $n<0, m \geq 0$. Consequently, $P_{F_{n}, F_{n+m}}=r_{1}^{-m} z^{m} P_{F_{n}}$ and $P_{F_{n}, F_{n-m}}=r_{1}^{-m}\left(z^{*}\right)^{m} P_{F_{n}}$. Hence, $P_{F_{n}, F_{k}} \in \mathcal{I}$ for all $n, k \leq-1$. Moreover, $P_{F_{n}, E_{k}}=r_{1}^{n} z^{m} P_{F_{n}}$ and so $P_{F_{n}, E_{k}} \in \mathcal{I}$ for $k \geq 0, n \leq-1$. In fact, it can be easily verified that, $P_{B_{j}, B_{i}}^{*}=P_{B_{i}, B_{j}}$. This would mean that $P_{E_{k}, F_{n}}$ also belong to $\mathcal{I}$. 
Similar calculations show that $P_{G_{n}, G_{n+m}}=r_{2}^{-m} P_{G_{n+m}} z^{m} P_{G_{n}}$ for $n+m<0$ and $P_{G_{n}, G_{n-m}}=$ $r_{2}^{-m} P_{G_{n-m}}\left(z^{*}\right)^{m} P_{G_{n}}$ for $n<0, m \geq 0$. Collectively, these imply that $P_{G_{n}, G_{k}} \in \mathcal{I}$ for all $n, k \leq-1$. Also $P_{G_{n}, E_{n+m}}=r_{2}^{m} P_{E_{n+m}} z^{m} P_{G_{n}}$ for $n+m \geq 0$. Hence $P_{G_{n}, E_{k}}$ and $P_{E_{k}, G_{n}}$ belong to $\in \mathcal{I}$ for all $k \geq 0, n \leq-1$.

Finally, we notice that $P_{G_{n}, F_{-m}}=r_{1}^{-m} r_{2}^{n} P_{F_{-m}}\left(z^{*}\right)^{m} P_{E_{0}} z^{n} P_{G_{n}}$, which shows that the operators $P_{G_{n}, F_{k}} \in \mathcal{I}$ for all $n, k \leq-1$.

Consider now finite rank operators which are finite linear combinations of the one-dimensional $P_{B_{i}, B_{j}}$ for $B_{i}, B_{j}$ in the basis for $H$. It is a simple exercise in functional analysis to show that all compact operators are norm limits of such finite rank operators.

To describe the structure of $C^{*}(z)$ we need to understand the commutative quotient $C^{*}(z) / \mathcal{K}$. For the case of quantum pair of pants that structure and the idea of proof is very similar to the case of quantum annulus.

Below we will show that the $C^{*}$-algebra $C^{*}(z)$ contains some infinite-dimensional projections. Those are obtained from the spectrum of $z z^{*}$. While tedious, the computation of the spectrum of $z z^{*}$ is fairly straightforward and it amounts to studying a (multi parameter) system of two step difference equations with constant coefficients. The results of the computations are presented in the next three theorems.

We start with the computation of the pure-point spectrum.

Theorem 3.11. The operator $z z^{*}$ has three eigenvalues: 1 with eigenspace $\operatorname{span}\left\{E_{n}\right\}_{n \geq 0}, r_{1}^{2}$ with eigenspace $\operatorname{span}\left\{F_{n}\right\}_{n<0}$, and the simple eigenvalue $\frac{r_{1}^{2}\left(a^{2}-r_{2}^{2}-r_{1}^{2}\right)}{a^{2}-r_{1}^{2}}$.

Proof. We study $\left(z z^{*}-\lambda\right) \varphi=0$. Using Lemma 3.3 we get the following system of equations

$$
\begin{array}{lr}
(1-\lambda) e_{n}=0 & \text { for } \quad n \geq 1, \\
\left(r_{1}^{2}+r_{2}^{2}-\lambda\right) e_{0}+a r_{2} g_{-1}=0, & \\
\left(r_{1}^{2}-\lambda\right) f_{n}=0 & \text { for } \quad n \leq-1, \\
a r_{2} g_{n+1}+\left(a^{2}+r_{2}^{2}-\lambda\right) g_{n}+a r_{2} g_{n-1}=0 & \text { for } \quad n \leq-2, \\
a r_{2} e_{0}+\left(a^{2}+r_{2}^{2}-\lambda\right) g_{-1}+a r_{2} g_{-2}=0 . &
\end{array}
$$

The first and the third equations in this system yield the eigenvalues $1, r_{1}^{2}$ and the eigenspaces $\operatorname{span}\left\{E_{n}\right\}_{n \geq 0}, \operatorname{span}\left\{F_{n}\right\}_{n<0}$ respectively. For the fourth equation, which is a two step linear recurrence with constant coefficients, the characteristic equation is

$$
a r_{2} x^{2}+\left(r_{2}^{2}+a^{2}-\lambda\right) x+a r_{2}=0 .
$$

The discriminant $\Delta$ of this equation is

$$
\Delta=\left(r_{2}^{2}+a^{2}-\lambda\right)^{2}-4 a^{2} r_{2}^{2}=\left(\left(a-r_{2}\right)^{2}-\lambda\right)\left(\left(a+r_{2}\right)^{2}-\lambda\right),
$$

and the roots are

$$
x_{ \pm}=\frac{\lambda-r_{2}^{2}-a^{2} \pm \sqrt{\Delta}}{2 a r_{2}} .
$$

Thus, the formal solution to the homogeneous equation is

$$
g_{n}=c_{+} x_{+}^{n+1}+c_{-} x_{-}^{n+1},
$$

where $c_{+}$and $c_{-}$are arbitrary constants. Notice that if we adopt the convention $g_{0}:=e_{0}$ in the last equation of (3.4), then this formula holds for $n \leq 0$. 
There are three separate cases to consider: $0<\lambda<\left(r_{2}-a\right)^{2},\left(r_{2}-a\right)^{2} \leq \lambda \leq\left(r_{2}+a\right)^{2}$ and $\left(r_{2}+a\right)^{2}<\lambda<1$. If $0<\lambda<\left(r_{2}-a\right)^{2}$ we notice the following facts about the solutions. Since $\lambda<\left(r_{2}-a\right)^{2}=r_{2}^{2}+a^{2}-2 a r_{2}$ and both $a$ and $r_{2}$ are positive, we have $\lambda-r_{2}^{2}-a^{2}<0$, and since $\Delta>0$, we have that $x_{-}<0$. Also notice that $x_{+} x_{-}=1$ from which it follows that $x_{+}<0$. Since $x_{-}<x_{+}$we have $x_{-}<-1$ and $-1<x_{+}<0$. Thus, $\left|x_{-}\right|>1$ and $\left|x_{+}\right|<1$.

Since we need $g_{n} \in \ell^{2}\left(\mathbb{Z}_{<0}\right)$, and since $\left|x_{+}\right|<1$, we must have $c_{+}=0$. In particular this implies that $g_{0}=x_{-} g_{-1}$. The second equation of the system (3.4) then becomes

$$
\left(\left(r_{1}^{2}+r_{2}^{2}-\lambda\right) x_{-}+a r_{2}\right) g_{-1}=0 .
$$

If $\left(r_{1}^{2}+r_{2}^{2}-\lambda\right) x_{-}+a r_{2}=0$, then using the relation $x_{+} x_{-}=1$ we obtain

$$
x_{+}=\frac{\lambda-r_{1}^{2}-r_{2}^{2}}{a r_{2}} .
$$

On the other hand we also have

$$
x_{+}=\frac{\lambda-a^{2}-r_{2}^{2}+\sqrt{\Delta}}{a r_{2}} .
$$

Setting these equal to each other and solving for $\Delta$ we get $\Delta=\left(\lambda-2 r_{1}^{2}-r_{2}^{2}+a^{2}\right)^{2}$. Solving for $\lambda$ yields

$$
\lambda=r_{1}^{2}\left(1-\frac{r_{1}^{2}}{a^{2}-r_{1}^{2}}\right)=\frac{r_{1}^{2}\left(a^{2}-r_{2}^{2}-r_{1}^{2}\right)}{a^{2}-r_{1}^{2}} .
$$

First notice that due to the conditions on $a, r_{1}$ and $r_{2}$ we have that $\lambda>0$ and $\lambda<r_{1}^{2}$ as $r_{1}^{2} /\left(a^{2}-r_{1}^{2}\right)>0$. Therefore this $\lambda$ is in the interval $\left(0,\left(r_{2}-a\right)^{2}\right)$, and $c_{-}=g_{-1}$ is arbitrary. Consequently $\lambda=\frac{r_{1}^{2}\left(a^{2}-r_{2}^{2}-r_{1}^{2}\right)}{a^{2}-r_{1}^{2}}$ is a simple eigenvalue with an eigenvector

$$
\varphi_{\lambda}=\sum_{n=-\infty}^{0} x_{-}^{n+1} G_{n} .
$$

In the case $\left(r_{2}+a\right)^{2}<\lambda<1$, we have $\lambda-r_{2}^{2}-a^{2}=\lambda-\left(r_{2}+a\right)^{2}+2 r_{2} a>0, \Delta>0$ and hence $x_{+}>0$. Again from $x_{+} x_{-}=1$ we see that $x_{+}>1$ and $0<x_{-}<1$. Consequently, in equation (3.5) we must have $c_{-}=0$, which then implies that $g_{0}=x_{+} g_{-1}$. The second equation of (3.4) then becomes

$$
\left(\left(r_{1}^{2}+r_{2}^{2}-\lambda\right) x_{+}+a r_{2}\right) g_{-1}=0 .
$$

Suppose there is $\lambda$ such that $x_{+}\left(r_{1}^{2}+r_{2}^{2}-\lambda\right)+a r_{2}=0$, which would then imply that

$$
x_{+}=\frac{a r_{2}}{\lambda-r_{1}^{2}-r_{2}^{2}}
$$

and, since $x_{+}>1$, we must have

$$
\lambda<r_{1}^{2}+a r_{2}+r_{2}^{2}<a^{2}+2 a r_{2}+r_{2}^{2}=\left(r_{2}+a\right)^{2} .
$$

This is a contradiction. Consequently $g_{n}=0$ for every $n$ and there are no eigenvectors in this case.

When $\left(r_{2}-a\right)^{2} \leq \lambda \leq\left(r_{2}+a\right)^{2}$ the discriminant $\Delta \leq 0$ and the two solutions $x_{+}$and $x_{-}$ are complex numbers conjugate to each other with absolute value equal to one. Since we need $g_{n} \in \ell^{2}\left(\mathbb{Z}_{<0}\right)$, equation (3.5) implies that $g_{n}=0$ and there are again no eigenvectors in this case. 
The second, and the most technical step in the calculation of the spectrum of $z z^{*}$ is the calculation of the inverse of $z z^{*}-\lambda$. Norm estimates of the inverse provide insight about the resolvent set of $z z^{*}$.

Lemma 3.12. The operator $\left(z z^{*}-\lambda\right)^{-1}$ is bounded for $\lambda$ not an eigenvalue and $\lambda \in\left(0,\left(r_{2}-\right.\right.$ $\left.a)^{2}\right) \cup\left(\left(r_{2}+a\right)^{2}, 1\right)$.

Proof. We study $\left(z z^{*}-\lambda\right) \varphi=\tilde{\varphi}$ using coordinates

$$
\varphi=\sum_{n=0}^{\infty} e_{n} E_{n}+\sum_{n=-\infty}^{-1}\left(f_{n} F_{n}+g_{n} G_{n}\right) \quad \text { and } \quad \tilde{\varphi}=\sum_{n=0}^{\infty} \tilde{e}_{n} E_{n}+\sum_{n=-\infty}^{-1}\left(\tilde{f}_{n} F_{n}+\tilde{g}_{n} G_{n}\right) .
$$

Using Lemma 3.3 we get the following system of equations

$$
\begin{array}{lrl}
(1-\lambda) e_{n}=\tilde{e}_{n} & \text { for } n \geq 1, \\
\left(r_{1}^{2}+r_{2}^{2}-\lambda\right) g_{0}+a r_{2} g_{-1}=\tilde{g}_{0}, & \\
\left(r_{1}^{2}-\lambda\right) f_{n}=\tilde{f}_{n} & \text { for } n \leq-1, \\
a r_{2} g_{n+1}+\left(a^{2}+r_{2}^{2}-\lambda\right) g_{n}+a r_{2} g_{n-1}=\tilde{g}_{n} & \text { for } n \leq-1 .
\end{array}
$$

For the purpose of this proof we have introduced the notation $g_{0}:=e_{0}$ and $\tilde{g}_{0}:=\tilde{e}_{0}$.

The first and the third equations in system (3.6) can be solved directly

$$
\begin{array}{ll}
e_{n}=\frac{1}{1-\lambda} \tilde{e}_{n} \quad \text { for } \quad n \geq 1 \quad \text { and } \quad \lambda \neq 1, \\
f_{n}=\frac{1}{r_{1}^{2}-\lambda} \tilde{f}_{n} \quad \text { for } \quad n \leq-1 \quad \text { and } \quad \lambda \neq r_{1}^{2} .
\end{array}
$$

As for the fourth equation in system (3.6), we start with the case $0<\lambda<\left(r_{2}-a\right)^{2}$. From the proof of Theorem 3.11 we have that $\left|x_{-}\right|>1$ and $\left|x_{+}\right|<1$, where $x_{ \pm}=\frac{\lambda-r_{2}^{2}-a^{2} \pm \sqrt{\Delta}}{2 a r_{2}}$ are the solution of the characteristic equation $a r_{2} x^{2}+\left(r_{2}^{2}+a^{2}-\lambda\right) x+a r_{2}=0$ with the discriminant $\Delta=\left(\left(a-r_{2}\right)^{2}-\lambda\right)\left(\left(a+r_{2}\right)^{2}-\lambda\right)$.

We use the variation of parameters technique to solve this equation. For the homogeneous component of the solution, we use (3.5) to obtain $g_{n}=c_{+} x_{+}^{n+1}+c_{-} x_{-}^{n+1}$ with $c_{+}$and $c_{-}$being constants to be determined. We look for the the solutions of the non-homogeneous equation in the form: $g_{n}=A_{n} x_{+}^{n+1}+B_{n} x_{-}^{n+1}$. Then the standard trick is to assume the first equation below to obtain the following system

$$
\begin{array}{ll}
\left(A_{n}-A_{n-1}\right) x_{+}^{n+1}+\left(B_{n}-B_{n-1}\right) x_{-}^{n+1}=0 & \text { for } \quad n \leq 0, \\
\left(A_{n}-A_{n-1}\right) x_{+}^{n}+\left(B_{n}-B_{n-1}\right) x_{-}^{n}=\frac{-\tilde{g}_{n}}{a r_{2}} & \text { for } \quad n \leq-1 .
\end{array}
$$

In particular,

$$
\left(A_{0}-A_{-1}\right) x_{+}+\left(B_{0}-B_{-1}\right) x_{-}=0 .
$$

The solution of the above system is

$$
A_{n}-A_{n-1}=\frac{\tilde{g}_{n} x_{-}^{n+1}}{\sqrt{\Delta}}, \quad B_{n}-B_{n-1}=\frac{-\tilde{g}_{n} x_{+}^{n+1}}{\sqrt{\Delta}} .
$$

In solving these difference equations we pay attention to square summability, making sure we only consider convergent expressions in powers of $x_{ \pm}$. This leads to the following special solution of the non-homogeneous equation

$$
A_{n}=\frac{1}{\sqrt{\Delta}} \sum_{j=-\infty}^{n} \tilde{g}_{j} x_{-}^{j+1} \quad \text { and } \quad B_{n}=\frac{1}{\sqrt{\Delta}} \sum_{j=n+1}^{-1} \tilde{g}_{j} x_{+}^{j+1}
$$


with $B_{-1}=0, n \leq-1$. Consequently the general solution is

$$
g_{n}=c_{-} x_{-}^{n+1}+\frac{1}{\sqrt{\Delta}}\left(\sum_{j=n+1}^{-1} \tilde{g}_{j} x_{+}^{j-n}+\sum_{j=-\infty}^{n} \tilde{g}_{j} x_{-}^{j-n}\right)
$$

for $n<-1$, since we want $g_{n} \in \ell^{2}\left(\mathbb{Z}_{<0}\right)$, and since $\left|x_{+}\right|<1$ we must have $c_{+}=0$. For $n=-1$ we get

$$
g_{-1}=c_{-}+\frac{1}{\sqrt{\Delta}} \sum_{j=-\infty}^{-1} \tilde{g}_{j} x_{-}^{j+1}
$$

and for $n=0$, using (3.7), we obtain

$$
g_{0}=c_{-} x_{-}+A_{0} x_{+}+B_{0} x_{-}=c_{-} x_{-}+A_{-1} x_{+}+B_{-1} x_{-}=c_{-} x_{-}+\frac{x_{+}}{\sqrt{\Delta}} \sum_{j=-\infty}^{-1} \tilde{g}_{j} x_{-}^{j+1} .
$$

Next we study the second equation of system (3.6). Substituting the formulas for $g_{0}$ and $g_{-1}$ we compute

$$
c_{-}=\frac{\tilde{g}_{0}}{x_{-}\left(r_{1}^{2}+r_{2}^{2}-\lambda\right)+a r_{2}}-\left(\frac{x_{+}\left(r_{1}^{2}+r_{2}^{2}-\lambda\right)+a r_{2}}{x_{-}\left(r_{1}^{2}+r_{2}^{2}-\lambda\right)+a r_{2}}\right) \frac{1}{\sqrt{\Delta}} \sum_{j=-\infty}^{-1} \tilde{g}_{j} x_{-}^{j+1} .
$$

The above formulas give the unique solution of the equation $\left(z z^{*}-\lambda\right) \varphi=\tilde{\varphi}$, and hence define the inverse operator $\left(z z^{*}-\lambda\right)^{-1}$. We need to verify that this operator is bounded. So we first define the following operator

$$
Q \tilde{g}=\sum_{n=-\infty}^{0}\left(\frac{\tilde{g}_{0}}{x_{-}\left(r_{1}^{2}+r_{2}^{2}-\lambda\right)+a r_{2}}-\left(\frac{x_{+}\left(r_{1}^{2}+r_{2}^{2}-\lambda\right)+a r_{2}}{x_{-}\left(r_{1}^{2}+r_{2}^{2}-\lambda\right)+a r_{2}}\right) \frac{1}{\sqrt{\Delta}} \sum_{j=-\infty}^{-1} \tilde{g}_{j} x_{-}^{j+1}\right) x_{-}^{n+1} G_{n} .
$$

Notice that, since $\left|x_{-}\right|>1, Q$ is a bounded operator taking $\ell^{2}\left(\mathbb{Z}_{\leq 0}\right)$ to itself. Here we have used the notation $G_{0}:=E_{0}$. Additionally we will need the following four operators, written in components:

$$
\begin{aligned}
& \left(L_{1} g\right)_{n}=\frac{1}{\sqrt{\Delta}} \sum_{j=-\infty}^{-1} x_{-}^{j-n} \chi\left(\frac{j}{n}\right) g_{j}: \ell^{2}\left(\mathbb{Z}_{<0}\right) \rightarrow \ell^{2}\left(\mathbb{Z}_{<0}\right), \\
& \left(L_{2} g\right)_{n}=\frac{1}{\sqrt{\Delta}} \sum_{j=-\infty}^{-1} x_{+}^{j-n} \chi\left(\frac{n+1}{j}\right) g_{j}: \ell^{2}\left(\mathbb{Z}_{<0}\right) \rightarrow \ell^{2}\left(\mathbb{Z}_{<0}\right), \\
& \left(L_{3} g\right)_{n}=\frac{1}{\sqrt{\Delta}} \sum_{j=-\infty}^{-1} x_{+}^{j-n} \chi\left(\frac{j}{n}\right) g_{j}: \ell^{2}\left(\mathbb{Z}_{<0}\right) \rightarrow \ell^{2}\left(\mathbb{Z}_{<0}\right), \\
& \left(L_{4} g\right)_{n}=\frac{1}{\sqrt{\Delta}} \sum_{j=-\infty}^{-1} x_{-}^{j-n} \chi\left(\frac{n+1}{j}\right) g_{j}: \ell^{2}\left(\mathbb{Z}_{<0}\right) \rightarrow \ell^{2}\left(\mathbb{Z}_{<0}\right) .
\end{aligned}
$$

Using these operators we write

$$
g_{n}=(Q \tilde{g})_{n}+\left(L_{1} \tilde{g}\right)_{n}+\left(L_{2} \tilde{g}\right)_{n} \quad \text { for } \quad n \leq-1
$$


and

$$
g_{0}=(Q \tilde{g})_{0}+x_{+}\left(L_{1} \tilde{g}\right)_{-1} .
$$

Then we use Lemma 3.6 (Schur-Young inequality) to estimate the norms of $L_{1}$ and $L_{2}$. We have

$$
\begin{aligned}
\left\|L_{1}\right\|^{2} & \leq \frac{1}{\Delta}\left(\sup _{n \leq-1}\left|x_{-}\right|^{-n} \sum_{j=-\infty}^{n}\left|x_{-}\right|^{j}\right)\left(\sup _{j \leq-1}\left|x_{-}\right|^{j} \sum_{n=j}^{-1}\left|x_{-}\right|^{-n}\right) \\
& =\frac{1}{\Delta}\left(\frac{1}{1-\left|x_{-}\right|^{-1}}\right)\left(\sup _{j \leq-1} \frac{1-\left|x_{-}\right|^{j}}{1-\left|x_{-}\right|^{-1}}\right)=\frac{1}{\Delta} \frac{1}{\left(1-\left|x_{-}\right|^{-1}\right)^{2}} .
\end{aligned}
$$

The computation of norm of $L_{2}$ is similar. Therefore $\left(z z^{*}-\lambda\right)^{-1}$ is bounded for $0<\lambda<\left(r_{2}-a\right)^{2}$.

The other case is when $\left(r_{2}+a\right)^{2}<\lambda<1$. From the proof of Theorem 3.11 we have that $x_{+}>1$ and $x_{-}<1$. Again using variation of parameters, we see that the particular solution of system (3.8) is given by:

$$
B_{n}=\frac{-1}{\sqrt{\Delta}} \sum_{j=-\infty}^{n} \tilde{g}_{j} x_{+}^{j+1} \quad \text { and } \quad A_{n}=\frac{-1}{\sqrt{\Delta}} \sum_{j=n+1}^{-1} \tilde{g}_{j} x_{-}^{j+1}
$$

with $A_{-1}=0, n \leq-1$. Consequently the general solution is

$$
g_{n}=c_{+} x_{+}^{n+1}-\frac{1}{\sqrt{\Delta}}\left(\sum_{j=n+1}^{-1} \tilde{g}_{j} x_{-}^{j-n}+\sum_{j=-\infty}^{n} \tilde{g}_{j} x_{+}^{j-n}\right) \quad \text { for } \quad n \leq-1 .
$$

Since we require $g_{n} \in \ell^{2}\left(\mathbb{Z}_{<0}\right)$, we must have $c_{-}=0$. For $n=-1$ we obtain

$$
g_{-1}=c_{+}-\frac{1}{\sqrt{\Delta}} \sum_{j=-\infty}^{-1} \tilde{g}_{j} x_{+}^{j+1}
$$

and for $n=0$, using (3.7), we have

$$
g_{0}=c_{+} x_{+}+A_{0} x_{+}+B_{0} x_{-}=c_{+} x_{+}+A_{-1} x_{+}+B_{-1} x_{-}=c_{+} x_{+}-\frac{x_{-}}{\sqrt{\Delta}} \sum_{j=-\infty}^{-1} \tilde{g}_{j} x_{+}^{j+1} .
$$

Substituting the formulas for $g_{0}$ and $g_{-1}$ into the second equation of system (3.6) we compute $c_{+}$

$$
c_{+}=\frac{\tilde{g}_{0}}{x_{+}\left(r_{1}^{2}+r_{2}^{2}-\lambda\right)+a r_{2}}+\left(\frac{x_{-}\left(r_{1}^{2}+r_{2}^{2}-\lambda\right)+a r_{2}}{x_{+}\left(r_{1}^{2}+r_{2}^{2}-\lambda\right)+a r_{2}}\right) \frac{1}{\sqrt{\Delta}} \sum_{j=-\infty}^{-1} \tilde{g}_{j} x_{+}^{j+1} .
$$

The formulas above define the inverse operator $\left(z z^{*}-\lambda\right)^{-1}$. To verify that this operator is bounded we define the following operator

$$
R \tilde{g}=\sum_{n=-\infty}^{0}\left(\frac{\tilde{g}_{0}}{x_{+}\left(r_{1}^{2}+r_{2}^{2}-\lambda\right)+a r_{2}}-\left(\frac{x_{-}\left(r_{1}^{2}+r_{2}^{2}-\lambda\right)+a r_{2}}{x_{+}\left(r_{1}^{2}+r_{2}^{2}-\lambda\right)+a r_{2}}\right) \frac{1}{\sqrt{\Delta}} \sum_{j=-\infty}^{-1} \tilde{g}_{j} x_{+}^{j+1}\right) x_{+}^{n+1} G_{n} .
$$

It is easy to see that $R$ is a bounded operator taking $\ell^{2}\left(\mathbb{Z}_{\leq 0}\right)$ to itself. Then we can write

$$
g_{n}=(R \tilde{g})_{n}-\left(L_{3} \tilde{g}\right)_{n}-\left(L_{4} \tilde{g}\right)_{n} \quad \text { for } \quad n \leq-1
$$


and

$$
g_{0}=(R \tilde{g})_{0}-x_{-}\left(L_{3} \tilde{g}\right)_{-1} .
$$

We use Lemma 3.6 to estimate the norms of $L_{3}$ and $L_{4}$, in a manner similar to $L_{1}$. We omit the repetitive details. This shows that $\left(z z^{*}-\lambda\right)^{-1}$ is bounded for $\left(r_{2}+a\right)^{2}<\lambda<1$.

In the theorem below, we will see that the interval $\left[\left(r_{2}-a\right)^{2},\left(r_{2}+a\right)^{2}\right]$ is the continuous part of the spectrum of $z z^{*}$, completing its full description.

Theorem 3.13. The spectrum of $z z^{*}$ is

$$
\sigma\left(z z^{*}\right)=\left\{\frac{r_{1}^{2}\left(a^{2}-r_{2}^{2}-r_{1}^{2}\right)}{a^{2}-r_{1}^{2}}\right\} \cup\left\{r_{1}^{2}\right\} \cup\left[\left(r_{2}-a\right)^{2},\left(r_{2}+a\right)^{2}\right] \cup\{1\} .
$$

Proof. Since $z z^{*}$ is a positive operator with norm 1, its spectrum must be a closed subset of the interval $[0,1]$. In Theorem 3.11 we computed the pure point spectrum of $z z^{*}$ while Lemma 3.12 identified intervals belonging to the resolvent set of $z z^{*}$. So it remains to analyze the interval $\left[\left(r_{2}-a\right)^{2},\left(r_{2}+a\right)^{2}\right]$. We will show that if $\lambda \in\left(\left(r_{2}-a\right)^{2},\left(r_{2}+a\right)^{2}\right)$ then $\operatorname{Ran}\left(z z^{*}-\lambda\right)$, the range of $\left(z z^{*}-\lambda\right)$, is not all of $H$.

In the notation of system (3.6) consider $\left(z z^{*}-\lambda\right) \varphi=\tilde{\varphi}$ with $\tilde{g}_{n}=0$ for $n \leq-1$ but $\tilde{g}_{0}=\tilde{e}_{0} \neq 0$. This leads to the equation

$$
a r_{2} g_{n+1}+\left(a^{2}+r_{2}^{2}-\lambda\right) g_{n}+a r_{2} g_{n-1}=0 \quad \text { for } \quad n \leq-1,
$$

with the general solution $g_{n}=c_{+} x_{+}^{n+1}+c_{-} x_{-}^{n+1}$. Since for $\left(r_{2}-a\right)^{2} \leq \lambda \leq\left(r_{2}+a\right)^{2}$, the two numbers $x_{+}$and $x_{-}$are complex conjugates each with magnitude one; which follows from the arguments in the last part of Theorem 3.11; we must have $g_{n}=0$ for $n \leq 0$ for $\varphi$ to be in $H$. This however contradicts the second equation of (3.6) with $\tilde{g}_{0}=\tilde{e}_{0} \neq 0$. Consequently there is no $\varphi \in H$ satisfying $\left(z z^{*}-\lambda\right) \varphi=\tilde{\varphi}$ for such $\tilde{\varphi}$ and $\operatorname{Ran}\left(z z^{*}-\lambda\right)$ is not $H$.

Let $P_{E}, P_{F}$, and $P_{G}$ be the orthogonal projections onto the infinite-dimensional span of $\left\{E_{n}\right\}_{n \geq 0},\left\{F_{n}\right\}_{n<0}$ and $\left\{G_{n}\right\}_{n<0}$ respectively.

Proposition 3.14. The projections $P_{E}, P_{F}$, and $P_{G}$ belong to the noncommutative pair of pants, i.e., they are all in $C^{*}(z)$.

Proof. Since Lemma 3.12 implies that $r_{1}^{2}$ is an isolated eigenvalue of $z z^{*}$, there exists a continuous real valued function $f$ so that $f\left(r_{1}^{2}\right)=1$ and $f$ is zero on the rest of the spectrum of $z z^{*}$. By functional calculus we have $f\left(z z^{*}\right)=P_{F}$ and so $P_{F} \in C^{*}(z)$. Similarly, since 1 is an isolated eigenvalue and we already know that $P_{E_{0}} \in C^{*}(z)$ we get $P_{E} \in C^{*}(z)$ as well. Since $P_{G}=I-P_{E}-P_{F}$, it then follows that $P_{G} \in C^{*}(z)$.

We can decompose the Hilbert space $H$ into $H \cong H_{E} \oplus H_{F} \oplus H_{G}$, where $H_{E} \cong \ell^{2}\left(\mathbb{Z}_{\geq 0}\right)$, $H_{F} \cong \ell^{2}\left(\mathbb{Z}_{<0}\right)$, and $H_{G} \cong \ell^{2}\left(\mathbb{Z}_{<0}\right)$ are the Hilbert spaces with basis elements $E_{n}, F_{n}$, and $G_{n}$ respectively. Since $\ell^{2}\left(\mathbb{Z}_{\geq 0}\right)$ is a subspace of $\ell^{2}(\mathbb{Z})$, which can be identified with $L^{2}\left(S^{1}\right)$ via the Fourier transform, we can view $H_{E}$ as a subspace of $L^{2}\left(S^{1}\right)$. More precisely, if $B_{n}=\zeta^{n}, n \in \mathbb{Z}$ is the standard basis in $L^{2}\left(S^{1}\right)$, then $H_{E}$ is identified with the $\operatorname{subspace} \operatorname{span}\left\{B_{n}\right\}_{n \geq 0}$ via

$$
E_{n} \mapsto B_{n} .
$$

Define $P_{\geq 0}: L^{2}\left(S^{1}\right) \rightarrow H_{E}$ to be the projection onto $H_{E}$. For a $\varphi \in C\left(S^{1}\right)$ we define $T_{E}(\varphi): H_{E} \rightarrow H_{E}$ by $T_{E}(\varphi)=P_{\geq 0} M(\varphi)$ where $M(\varphi): L^{2}\left(S^{1}\right) \rightarrow L^{2}\left(S^{1}\right)$ is the multiplication operator by $\varphi$. In particular for $\varphi(\zeta)=\zeta$ we have

$$
T_{E}(\zeta) E_{n}=E_{n+1},
$$

the unilateral shift. 
Similarly we identify $H_{F}$ and $H_{G}$ with the subspace $\operatorname{span}\left\{B_{n}\right\}_{n<0}$ in $L^{2}\left(S^{1}\right)$, and let $P_{<0}$ : $L^{2}\left(S^{1}\right) \rightarrow H_{F}$ and $P_{<0}^{\prime}: L^{2}\left(S^{1}\right) \rightarrow H_{G}$ be the orthogonal projections onto $H_{F}$ and $H_{G}$ respectively. Then for a $\varphi \in C\left(S^{1}\right)$, we define $T_{F}(\varphi): H_{F} \rightarrow H_{F}$ by $T_{F}(\varphi)=P_{<0} M(\varphi)$ and $T_{G}(\varphi): H_{G} \rightarrow H_{G}$ by $T_{G}(\varphi)=P_{<0}^{\prime} M(\varphi)$ respectively. We have

$$
T_{F}(\zeta) F_{n}=F_{n+1}, \quad T_{G}(\zeta) G_{n}=G_{n+1} \quad \text { for } \quad n<-1
$$

and

$$
T_{F}(\zeta) F_{-1}=0, \quad T_{G}(\zeta) G_{-1}=0 .
$$

The operators $T_{E}(\varphi), T_{F}(\varphi)$, and $T_{G}(\varphi)$ may be viewed as Toeplitz operators and they will be needed in proving the following result.

Theorem 3.15. The quotient $C^{*}(z) / \mathcal{K}$ is isomorphic to $C\left(S^{1}\right) \oplus C\left(S^{1}\right) \oplus C\left(S^{1}\right)$.

Proof. Using the above notation define

$$
T: C\left(S^{1}\right) \oplus C\left(S^{1}\right) \oplus C\left(S^{1}\right) \rightarrow C^{*}(z) / \mathcal{K}
$$

by

$$
T\left(\varphi_{1}, \varphi_{2}, \varphi_{3}\right)=T_{E}\left(\varphi_{1}\right) P_{E}+T_{F}\left(\varphi_{2}\right) P_{F}+T_{G}\left(\varphi_{3}\right) P_{G}+\mathcal{K}
$$

for continuous functions $\varphi_{1}, \varphi_{2}$, and $\varphi_{3}$ on the unit circle. To see that $T$ is well defined we need to show that $T\left(\varphi_{1}, \varphi_{2}, \varphi_{3}\right)$ is in $C^{*}(z)$. We showed that $P_{E} \in C^{*}(z)$, and notice that $T_{E}(\zeta) \in C^{*}(z)$ because $T_{E}(\zeta)=z P_{E}$. But Toeplitz operators $T_{E}(\varphi)$ can be uniformly approximated by polynomials in $T_{E}(\zeta)$ and its adjoint, and so $T_{E}\left(\varphi_{1}\right) P_{E} \in C^{*}(z)$. Similar arguments work for $T_{F}\left(\varphi_{2}\right) P_{F}$ and $T_{G}\left(\varphi_{3}\right) P_{G}$.

We verify that $T$ in (3.12) is a isomorphism between the two algebras. First notice that equation (3.12) implies that $T$ is continuous and linear. Next we show that the kernel of $T$ is trivial. Consider the equation $T\left(\varphi_{1}, \varphi_{2}, \varphi_{3}\right)=0$ implying that $T_{E}\left(\varphi_{1}\right) P_{E}+T_{F}\left(\varphi_{2}\right) P_{F}+T_{G}\left(\varphi_{3}\right) P_{G}$ is compact. Since $P_{E}, P_{F}$ and $P_{G}$ are orthogonal, $T_{E}\left(\varphi_{1}\right) P_{E}, T_{F}\left(\varphi_{2}\right) P_{F}$ and $T_{G}\left(\varphi_{3}\right) P_{G}$ must be compact, and consequently $T_{E}\left(\varphi_{1}\right): H_{E} \rightarrow H_{E}, T_{F}\left(\varphi_{2}\right): H_{F} \rightarrow H_{F}$, and $T_{G}\left(\varphi_{3}\right): H_{G} \rightarrow H_{G}$ are compact. By the proof of Theorem 2.3, it follows that $\varphi_{1}=\varphi_{2}=\varphi_{3}=0$ and thus the kernel of $T$ is trivial.

Next we show that $T$ is a homomorphism of algebras. Consider the difference:

$$
\begin{aligned}
& T\left(\varphi_{1}, \varphi_{2}, \varphi_{3}\right) T\left(\psi_{1}, \psi_{2}, \psi_{3}\right)-T\left(\varphi_{1} \psi_{1}, \varphi_{2} \psi_{2}, \varphi_{3} \psi_{3}\right) \\
& \quad=\left(T_{E}\left(\varphi_{1}\right) T_{E}\left(\psi_{1}\right)-T_{E}\left(\varphi_{1} \psi_{1}\right)\right) P_{E} \\
& \quad+\left(T_{F}\left(\varphi_{2}\right) T_{F}\left(\psi_{2}\right)-T_{F}\left(\varphi_{2} \psi_{2}\right)\right) P_{F}+\left(T_{G}\left(\varphi_{3}\right) T_{G}\left(\psi_{3}\right)-T_{G}\left(\varphi_{3} \psi_{3}\right)\right) P_{G} .
\end{aligned}
$$

Since $T_{E}, T_{F}$, and $T_{G}$ are Toeplitz operators, the proof of Theorem 2.3 implies that all three differences on the right hand side of equation (3.13) are compact operators. Thus $T$ is a homomorphism between the two algebras.

To show that the range of $T$ is dense we consider the difference $T\left(\zeta, r_{1} \zeta, r_{2} \zeta+a\right)-z$. Using formulas (3.9), (3.10), and (3.11) we get $T_{E}(\zeta) E_{n}=E_{n+1}$, for $n \geq 0, T_{F}\left(r_{1} \zeta\right) F_{n}=r_{1} F_{n+1}$, for $n<-1$, and $T_{G}\left(r_{2} \zeta+a\right) G_{n}=r_{2} G_{n+1}+a G_{n}$, for $n<-1$. Observe that $T\left(\zeta, r_{1} \zeta, r_{2} \zeta+a\right)-z$ is not zero on $F_{-1}$ and $G_{-1}$ only and hence it is a compact operator. Thus we have constructed functions $\varphi_{1}, \varphi_{2}$, and $\varphi_{3}$ such that $T\left(\varphi_{1}, \varphi_{2}, \varphi_{3}\right)=z$ in $C^{*}(z) / \mathcal{K}$. Since the $C^{*}$-algebra $C^{*}(z) / \mathcal{K}$ is generated by (the class of) $z$, the range of $T$ is dense and since the range of a $C^{*}$-morphism must be closed, $T$ is an isomorphism of algebras. This completes the proof. 
Note that from Theorem 3.15 we get a short exact sequence

$$
0 \rightarrow \mathcal{K} \rightarrow C^{*}(z) \rightarrow C\left(S^{1}\right) \oplus C\left(S^{1}\right) \oplus C\left(S^{1}\right) \rightarrow 0 .
$$

We can compare this to the short exact sequence for the classical pair of pants

$$
0 \rightarrow C_{0}\left(P P_{\left(a, r_{1}, r_{2}\right)}\right) \rightarrow C\left(P P_{\left(a, r_{1}, r_{2}\right)}\right) \rightarrow C\left(S^{1}\right) \oplus C\left(S^{1}\right) \oplus C\left(S^{1}\right) \rightarrow 0
$$

where $C_{0}\left(P P_{\left(a, r_{1}, r_{2}\right)}\right)$ are the continuous functions on the pair of pants that vanish on the boundary.

\section{References}

[1] Abrahamse M.B., Toeplitz operators in multiply connected regions, Amer. J. Math. 96 (1974), $261-297$.

[2] Abrahamse M.B., Douglas R.G., Operators on multiply connected domains, Proc. Roy. Irish Acad. Sect. A 74 (1974), 135-141.

[3] Ahlfors L.V., Complex analysis. An introduction to the theory of analytic functions of one complex variable, 3rd ed., International Series in Pure and Applied Mathematics, McGraw-Hill Book Co., New York, 1978.

[4] Coburn L.A., Singular integral operators and Toeplitz operators on odd spheres, Indiana Univ. Math. J. 23 (1973), 433-439.

[5] Conway J.B., A course in operator theory, Graduate Studies in Mathematics, Vol. 21, Amer. Math. Soc., Providence, RI, 2000.

[6] Halmos P.R., Sunder V.S., Bounded integral operators on $L^{2}$ spaces, Ergebnisse der Mathematik und ihrer Grenzgebiete, Vol. 96, Springer-Verlag, Berlin - New York, 1978.

[7] Klimek S., Lesniewski A., Quantum Riemann surfaces. I. The unit disc, Comm. Math. Phys. 146 (1992), 103-122.

[8] Klimek S., Lesniewski A., Quantum Riemann surfaces. II. The discrete series, Lett. Math. Phys. 24 (1992), 125-139.

[9] Klimek S., Lesniewski A., A two-parameter quantum deformation of the unit disc, J. Funct. Anal. 115 (1993), 1-23.

[10] Klimek S., Lesniewski A., Quantum Riemann surfaces. III. The exceptional cases, Lett. Math. Phys. 32 (1994), 45-61.

[11] Klimek S., Lesniewski A., Quantum Riemann surfaces for arbitrary Planck's constant, J. Math. Phys. 37 (1996), 2157-2165.

[12] Klimek S., McBride M., D-bar operators on quantum domains, Math. Phys. Anal. Geom. 13 (2010), 357-390, arXiv:1001.2216.

[13] Klimek S., McBride M., A note on Dirac operators on the quantum punctured disk, SIGMA 6 (2010), 056, 12 pages, arXiv:1003.5618.

[14] Klimek S., McBride M., Classical limit of the d-bar operators on quantum domains, J. Math. Phys. 52 (2011), 093501, 16 pages, arXiv:1101.2645.

[15] Klimek S., McBride M., A note on gluing Dirac type operators on a mirror quantum two-sphere, SIGMA 10 (2014), 036, 15 pages, arXiv:1309.7096.

[16] Markushevich A.I., Theory of functions of a complex variable, Chelsea Publishing Co., New York, 2005. 